\title{
Patientenhotels in Deutschland Bislang keine Ergänzung zur stationären Versorgung
}

Unter dem wirtschaftlichen Druck, der auf deutschen Kliniken durch die Einführung der Fallpauschalen lastet, kann es sich im Einzelfall rechnen, ein Patientenhaus zu betreiben. Solche Häuser beziehungsweise Hotelstationen sind für Patienten gedacht, die in der letzten Phase ihres stationären Aufenthalts nur noch ein geringes Maß an medizinischer und pflegerischer Versorgung benötigen. Patientenhäuser bieten in aller Regel den Luxus eines 3-4-Sterne-Hotels. Für die Krankenhäuser lassen sich Einsparungen vor allem durch geringere Personal- und Bettenkosten erzielen. Dennoch betreiben hierzulande bislang nur sehr wenige Kliniken ein eigenes Patientenhotel. Vorreiter ist das Universitätsklinikum Mannheim, das seinen Service sowohl Privat- als auch GKV-Patienten zur Verfügung stellt.

Von so genannten Patientenhotels beziehungsweise Patientenhäusern versprechen sich Kliniken einen Wettbewerbsvorteil gegenüber anderen Einrichtungen sowie die Möglichkeit, Kosten zu senken. Die Ansätze unterscheiden sich allerdings zum Teil erheblich voneinander. Manche Krankenhäuser beschränken sich darauf, für Privatpatienten oder Selbstzahler Komfortstationen mit hotelähnlichem Ambiente und Wellnessbereich einzurichten. Andere wiederum haben eigenständige Patientenhäuser im Stile eines 3- oder 4-Sterne-Hotels auf dem Klinikgelände oder in unmittelbarer Nähe errichten lassen, in denen sie Patienten unterbringen, die nur noch ein geringes Maß an medizinischer Versorgung benötigen. Die Bettenzahlen variieren dabei von knapp einem Dutzend bis hin zu 120 Betten. In Einzelfällen profitieren auch Mitglieder der Gesetzlichen Krankenversicherung von dem Angebot. Einige Einrichtungen stehen zudem Angehörigen oder Tagungsgästen offen.

\section{Patientenhotels in Deutschland noch nicht etabliert}

Anders als in anderen Ländern, wie Schweden, den USA oder Australien, wo Patientenhotels längst als Qualitätsmerkmal für Kliniken gelten, haben sich derartige Low-Care-Einrichtungen in Deutschland allerdings noch keineswegs flächendeckend etabliert. Nach der anfänglichen Euphorie vor einigen Jahren ist es vielmehr wieder still um das Thema Patientenhotel geworden.

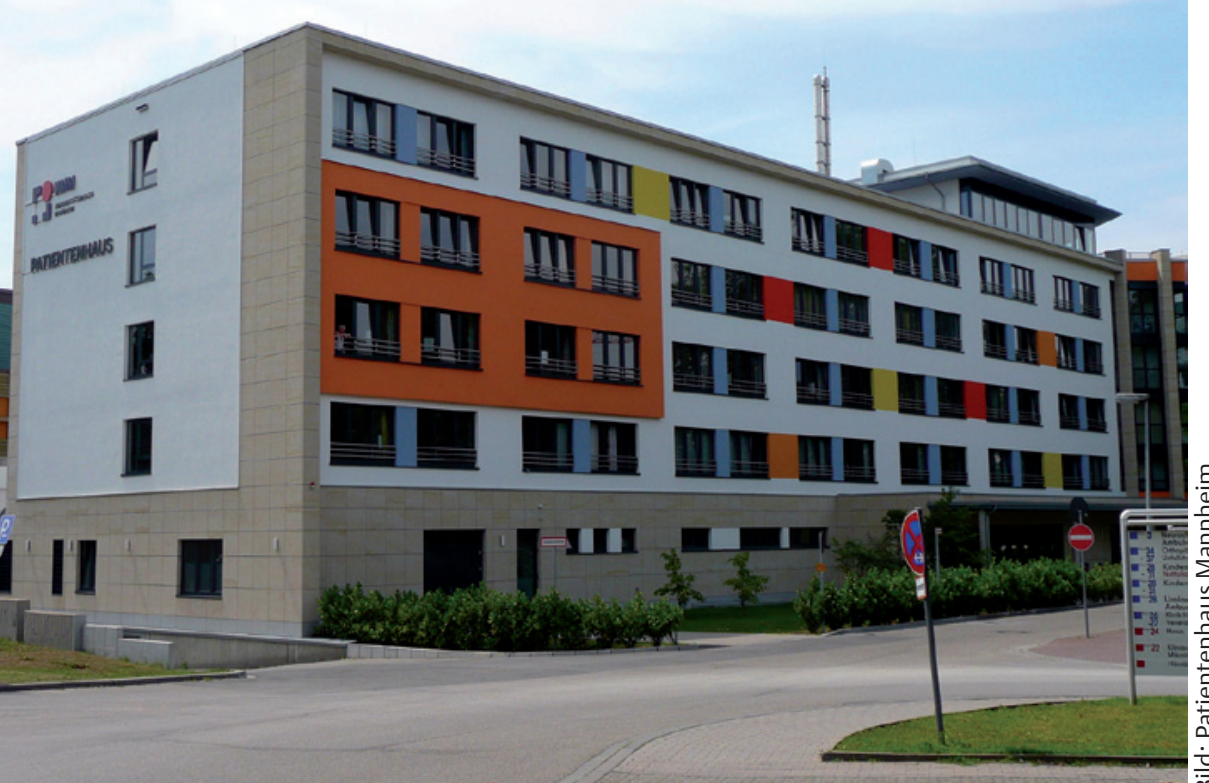

„In Schweden sind Patientenhotels längst integraler Bestandteil der Krankenhausversorgung“, erklärt Franz-Josef Richter, Geschäftsführer der Ahr Servita $\mathrm{GmbH}$, Oberhausen, einem Dienstleister für die Gesundheits- und Sozialwirtschaft. Etwa 50 solcher Einrichtungen gibt es dort inzwischen. Die Häuser stehen sowohl gesetzlich als auch privat versicherten $\mathrm{Pa}$ tienten gleichermaßen zur Verfügung. Die Vergütung der Versorgung in den Low-Care-Einrichtungen erfolgt über die DRG-Fallpauschalen. Bis zu 25\% aller stationär aufgenommenen Patienten - mit Ausnahme der Intensivpatienten - verbringen einen Teil ihres Klinikaufenthalts im Patientenhotel. „In Schweden ist die Bereitschaft einfach größer, sich auf neue Versorgungsmodelle einzulassen“, meint Richter. Hierzulande spreche hingegen wenig dafür, dass sich an der Zurückhaltung in nächster Zeit etwas ändern werde.

Den meisten deutschen Klinikbetreibern, die zunächst großes Interesse an der Errichtung eines Patientenhotels gezeigt hätten, habe letztlich der Mut zur Umsetzung gefehlt. „Man wollte lieber abwarten, bis es das irgendwo anders gibt“, sagt Richter.

\section{Zunächst hohe Investitionskosten vor Kosteneinsparung}

Nicht zu verkennen ist auch, dass trotz der zu erwartenden Kosteneinsparungen zunächst erhebliche Summen in die Errichtung eines eigenständigen Patientenhauses oder den Umbau eines bestehenden Gebäudes investiert werden müssen. 19,5 Millionen Euro sollte beispielsweise der Bau eines Patientenhotels mit 120 Zimmern auf dem Campus des Universitätsklinikums Lübeck kosten. Als Partner war die Maritim Hotelkette ausersehen. Der Baubeginn war für das Jahr 2008 geplant. Das Universitätsklinikum Kiel sollte 2 Jahre später ebenfalls ein eigenes Patientenhotel bekommen. Aus dem Vorhaben wurde dann allerdings nichts. Offiziell heißt es, Schwierigkeiten bei der Bettenplanung seien der Grund hierfür gewesen. Hinter vorgehaltener Hand wird indes gemunkelt, dass die hohen Investitionskosten Schuld am Scheitern des Projekts seien. 
Das Universitätsklinikum Mannheim (UKM) wiederum, das das bislang größte versichertenübergreifende Patientenhaus in Deutschland betreibt, hat rund 13 Millionen Euro in den Bau der Einrichtung gesteckt. Das Haus wurde im November 2008 auf dem Klinikgelände errichtet und bietet Platz für 119 Patienten. Einer Evaluationsstudie des Deutschen Krankenhausinstituts (DKI) zufolge könnten sich die Investitionskosten bei einer durchschnittlichen Mindestauslastung von $65 \%$ binnen 10 Jahren amortisiert haben. Derzeit liegt die durchschnittliche Belegungsquote bei über $80 \%$.

\section{Kosteneinsparung durch niedrigere Personalkosten}

Die Kosteneinsparungen ergeben sich in erster Linie durch niedrigere Personalkosten, da Servicetätigkeiten von Hotelfachpersonal kostengünstiger erbracht werden können als Pflegeleistungen. „Die Lohnkosten in diesen Bereichen sind bis zu $40 \%$ geringer als die von geprüften Pflegekräften“, schreibt Katrin Tränkner, Autorin der Studie des Buchs „Patientenhotels in Deutschland - Keine Zukunftsvision“. Hinzu kommen niedrigere Kosten für die Unterbringung in einem Patientenhotel. Ein Akutbett koste bei einem normalen Krankenhausaufenthalt am Tag 120-250 Euro, so Tränkner. „Die Unterbringung in Low-Care-Einrichtungen hingegen kostet pro Tag und Patient nur 60100 Euro.“

In Mannheim liegen die Tageskosten für die Pflege, Unterkunft und Verpflegung nach Schätzungen des DKI um 25-50\% unter denen, die auf einer Normalstation des Klinikums anfallen würden. Die Patienten, die von verschiedenen Akutstationen des UKM, wie der HNO-Abteilung, der Gynäkologie, der Augenheilkunde oder der Neurologie, ins Patientenhaus verlegt werden, verbringen in der LowCare-Einrichtung in aller Regel noch einige Tage, bevor sie nach Hause entlassen werden. 37 Vollzeitbeschäftigte, davon 14 examinierte Pflege- und 23 Servicekräfte, kümmern sich um die Rundumversorgung der Gäste. Über die Verlegung ins Patientenhaus entscheidet in jedem Einzelfall der behandelnde Arzt.

„Die Patienten bedürfen zwar noch einer medizinischen und pflegerischen Betreuung, sind aber bereits so mobil, dass sie ihr Essen beispielsweise im Restaurant einnehmen oder zur Visite in den Visiten- bereich des Patientenhotels gehen können“, erklärt Dr. Katja Goihl von der Allbau Medi Konsult GmbH, einem Unternehmen der Allbau-Gruppe in Berlin, die sich auf die Umsetzung, Beratung und Begleitung von Projekten in der Gesundheitsbranche spezialisiert hat. Die Medi Konsult ist für die wissenschaftliche Begleitung des Patientenhauses verantwortlich.

Da das Patientenhaus als interdisziplinäre Station des UKM betrieben wird, erfolgt die Abrechnung aller Leistungen automatisch über die Fallpauschalen. Somit können auch GKV-Patienten ohne Zuzahlungen in den Genuss des zusätzlichen Service kommen.

\section{Unterschiedliche Meinungen bei Krankenhausträgern und Kranken- kassen}

Nicht alle Krankenhausträger sind indes von den Vorteilen eines Patientenhauses überzeugt. „Sofern ein stationärer Aufenthalt in einem Krankenhaus medizinisch notwendig ist, sind aus unserer Sicht Patientenhotels nicht die geeignete Versorgungsform. Die akutstationären Voraussetzungen für eine qualitativ hochwertige Patientenversorgung müssen immer gegeben sein“, teilt Kerstin Kröning, Sprecherin der Helios Kliniken $\mathrm{GmbH}$, mit.

Auch bei den Krankenkassen gehen die Meinungen über Patientenhotels auseinander. „Hotelaufenthalte sind keine Alternative zu einer qualitativ hochwertigen Krankenhausbehandlung“, erklärt Udo Barske, Sprecher des AOK Bundesverbandes. Sie leisteten keinen Beitrag zu einer schnelleren Genesung und seien für die Krankenhausunternehmen lediglich eine Möglichkeit, zusätzliche Umsätze zu erzielen und die Anzahl der Behandlungsfälle zu steigern.

DAK-Vorstandschef Prof. Dr. h.c. Herbert Rebscher hingegen sieht in Patientenhotels eine Chance für Krankenhäuser, ihr Leistungsportfolio zu erweitern und einen strategischen Vorteil gegenüber anderen Häusern zu schaffen. „Es wird ja nicht teurer“, so Rebscher bei einer Befragung der Kostenträger durch Buchautorin Tränkner. Ähnlich positiv hatten sich auch die Barmer Ersatzkasse, die Techniker Krankenkasse sowie die AOK Schleswig-Holstein geäußert.

Ein knappes Drittel der 35 von Tränkner befragten Krankenkassen lehnte allerdings eine Verlegung von Versicherten in ein Patientenhaus angesichts der zumeist ohnehin kurzen Verweildauern grundsätzlich ab. Die meisten Krankenkassen bezweifeln zudem, dass mit dem Errichten und Betreiben eines Patientenhotels Kosten gesenkt werden können. Knapp $63 \%$ äußerten sich darüber hinaus skeptisch hinsichtlich der Sicherstellung der medizinischen Leistungen in einem Patientenhaus.

Richter hingegen ist der Ansicht, dass sich ein Patientenhotel rechnen kann. Je nach Größenordnung einer Klinik ließen sich pro Jahr Mehrerlöse bis zu sechsstelliger Höhe erzielen. Entscheidend bei der Konzeption der Komfortlösung sei allerdings nicht der geringe Pflegebedarf, sondern der Versicherungsstatus. „Deshalb haben wir uns auf die Zielgruppe der Privatpatienten konzentriert“, so Richter.



Korrespondenz Petra Spielberg Fachjournalistin für Gesundheitsund Sozialpolitik Wiesbaden/Brüssel Fax: 0611/98818512 p.spielberg@t-online.de 